\title{
Analysis of ribonucleotide reductase M2 mRNA levels in patient samples after GTI-2040 antisense drug treatment
}

\author{
AGNES JUHASZ ${ }^{1}$, AIKATERINI VASSILAKOS ${ }^{2}$, HELEN K. CHEW $^{3}$, DAVID GANDARA ${ }^{3}$ and YUN YEN ${ }^{1}$ \\ ${ }^{1}$ Department of Medical Oncology and Therapeutics Research, City of Hope Comprehensive Cancer Center, \\ 1500 E. Duarte Road, Duarte, CA 91010, USA; ${ }^{2}$ Lorus Therapeutics, 2 Meridian Road, Toronto, \\ Ontario M9W 4Z7, Canada; ${ }^{3}$ Division of Hematology/Oncology, University of California, \\ Davis Cancer Center, 4501 X Street, Sacramento, CA 95817, USA
}

Received October 3, 2005; Accepted November 30, 2005

\begin{abstract}
This study describes the development of a rapid and practical real-time RT-PCR method to quantify ribonucleotide reductase M2 (RRM2) mRNA in tumor and peripheral white blood cells (WBCs) from patients treated with GTI-2040, an antisense drug currently in clinical trials. In order to assess target down-regulation by GTI-2040, RRM2 mRNA expression levels were analyzed in pre- and post-treatment samples from a phase II clinical trial of GTI-2040 combined with capecitabine in patients with metastatic breast cancer. Target gene RRM2 mRNA levels were evaluated using quantitative RT-PCR method: real-time PCR (TaqMan ${ }^{\circledR}$ ) with fluorescein labeled probes on an ABI 7900HT instrument, with additional post-processing of the data to adjust for differences in total RNA in-put across the samples. Data are presented from a patient for whom both biopsy and PBMC samples were available, demonstrating applicability of this reproducible, highly sensitive real-time RT-PCR method for the detection and quantification of mRNAs for RRM2 in human WBC and tissue samples. By providing quantitative measurement of changes in target gene expression, this method may provide an opportunity to determine the correlation between target response to GTI-2040 antisense and clinical response in patients. Furthermore this assay may assess whether WBC samples are an appropriate surrogate tissue for approximating target down-regulation in the tumor.
\end{abstract}

\section{Introduction}

Mammalian ribonucleotide reductase (RR) catalyzes the reaction in which 2'-deoxyribonucleotides (dADP, dGDP, $\mathrm{dUDP}$ and $\mathrm{dCDP}$ ) are synthesized from the corresponding

Correspondence to: Dr Yun Yen, Division of Medical Oncology, 1500 E. Duarte Road, Duarte, CA 91010, USA

E-mail: yyen@coh.org

Key words: GTI-2040 antisense, real-time PCR, gene expression profiling, RRM1, RRM2, clinical trials ribonucleoside 5'-diphosphates (ADP, GDP, UDP and CDP). This step is the rate-limiting reaction in the production of 2'-deoxyribonucleoside 5'-triphosphates required for DNA replication (1). RR consists of two protein components. The RRM1 protein is a $160-\mathrm{kD}$ dimer that contains at least two different effector-binding sites. The RRM2 protein is a $78-\mathrm{kD}$ dimer that contains a non-heme iron that participates in the catalysis by forming an unusual free radical on the aromatic ring of a tyrosine residue. The mRNAs for the two components of RR are differentially expressed throughout the cell cycle. The level of RRM1 protein expression remains relatively stable throughout the cell cycle, with RRM2 only evident during late G1/early S phase, when DNA replication occurs. Regulation of RR activity is controlled by the amount of enzyme present in the cell and allosteric control mechanisms involving positive and negative effectors $(1,2)$. Ensuring proper function of RR is essential in the highly regulated process of cell replication and DNA repair. It has been shown that the elevated levels of RR expression and activity observed in a variety of tumor cells directly contribute to tumorigenic potential. Treatment of proliferating cells with RR inhibitors results in decreased pools of deoxyribonucleoside triphosphates that, over an extended period, lead to apoptosis (3-8). This effect is consistent with the hypothesis that RR is essential for tumor growth and proliferation.

The RRM2 protein appears to play a role in determining the malignant potential of cells via synergism with activated oncogenes with different functions and cellular locations. For example, anchorage-independent growth of cells transformed with $v$-fms, $v$-src, A-raf, v-fes, $c$-myc and ornithine decarboxylase was significantly enhanced when the RRM2 component of RR was overexpressed (9). In addition, altered RRM2 gene expression cooperates with ras in mechanisms of malignant progression. The overexpression of RRM2 protein results in significant increases in membrane-associated Raf-1 protein and mitogen-activating protein kinase-2 (MAPK) activity, thus implicating a major Ras pathway in the Ras/RRM2 synergism (10). Additionally, increased expression of RRM2 has been found to increase drug resistance in cancer cells. In contrast, a retrovirus vector containing the RRM2 sequence in an antisense orientation in these cells has been demonstrated to reverse such drug resistance in vitro (11). 
More recently, the effect of decreasing RRM2 levels on proliferation of tumor cells and tumor growth was examined in vitro and in vivo using siRNA molecules targeting the RRM2 gene. Ping Lin et al and Duxbury et al demonstrated the potential of siRNA targeting the M2 subunit of RR (12-14). siRNA-mediated inhibition of RRM2 expression sensitized colon cancer cells to cisplatin, Triapine ${ }^{\circledR}$ and hydroxyurea (12). RRM2 overexpression in pancreatic cancer cells has been associated with resistance to gemcitabine $(13,14)$. siRNA specific to RRM2 sensitized pancreatic cancer cells to gemcitabine and demonstrated cooperative antiproliferative, anti-tumor and antimetastatic activity in combination with gemcitabine against pancreatic adenocarcinoma cells and tumors both in vitro and in vivo, in animal models of human pancreatic cancer $(13,14)$. Taken together, the results of the above studies suggest that specific inhibition of the RRM2 protein in combination with chemotherapy can provide further antineoplastic benefit beyond the antiproliferative effects of RR inhibition.

Therapeutic agents, currently in use, that inhibit ribonucleotide reductase as part of their mechanism of action include hydroxyurea, gemcitabine and fludarabine. Gemcitabine and fludarabine are not specific inhibitors of ribonucleotide reductase and this non-specificity induces significant side effects that limit their effectiveness. GTI-2040 is a 20-mer phosphorothioate oligonucleotide that is complementary to RRM2 mRNA (15). An oligonucleotide, such as GTI-2040, directed against RRM2 mRNA, is very specific and works by inhibiting at the messenger RNA level, such that translation of the specific protein decreases. Thus, it is expected to be less toxic than conventional chemotherapeutic agents. Lorus Therapeutics, Inc. (Lorus) has tested GTI-2040 in a number of in vitro and in vivo models and results of these studies demonstrate that GTI-2040 is a selective and specific antitumor agent (16). GTI-2040 inhibits the growth of a number of cultured tumor cells in colony forming assays (15) and inhibits the growth of human colon, pancreas, liver, lung, breast, renal, ovarian, melanoma, brain glioblastoma-astrocytoma, prostate and cervical tumors in xenograft models (16). In addition, GTI-2040 dramatically increased the survival of mice in two experimental models of hematological malignancy (16). In this same study, GTI-2040 was effective in reducing lung metastases in mice injected with human malignant melanoma cells (16). The anti-tumor activity of GTI-2040 was found to be sequence specific, as scrambled and mismatched control oligonucleotides were ineffective. Although phosphorothioate oligonucleotides (PS-ODNs) have been found to have immune stimulatory functions leading to varying degrees of anti-tumor activity, it does not appear as though GTI-2040 functions via this mechanism, as the anti-tumor activity remained unchanged in severely immune compromised SCID beige mice (16).

The toxicities, pharmacokinetics and tissue distribution of GTI-2040 in both rats and monkeys were typical for the phosphorothioate oligonucleotide class of compounds (unpublished data) $(17,18)$. There were no apparent sequencespecific effects related to the interaction of GTI-2040 with RRM2 mRNA.

Clinical trials of GTI-2040 have been initiated in the United States by Lorus. Additional trials have been initiated in the
US and Canada by the National Cancer Institute (NCI) under the Cancer Therapy Evaluation Program (CTEP). The results of the first clinical trial for GTI-2040, Protocol GTI-2040-MI-1, A Phase I study of GTI-2040 given by continuous intravenous infusion in patients with advanced or metastatic solid tumors or lymphoma, were published recently in Annals of Oncology (19). This study determined that GTI-2040 has a good safety profile at the recommended phase II dose of $185.0 \mathrm{mg} / \mathrm{m}^{2} /$ day; although no objective responses were observed, disease stabilization in this subset of patients was encouraging for further development. A second clinical study, Protocol L01-1409, A Phase I/II study of GTI-2040 and capecitabine combination therapy in patients with advanced or metastatic renal cell carcinoma (mRCC), has recently been completed and final study reports are pending. In an interim analysis reported earlier (20), treatment was well tolerated in the 29 patients assessed, with few treatment-related toxicities other than those already known to occur with these drugs. At the recommended dose, 25 patients were evaluable for best response. Of these, 13 (52\%) had stable disease (SD) of median duration 4 months (range 2-10 months), with 23\% tumor reduction in the patient with the most sustained SD, and one durable partial response (PR) was observed with unidimensional tumor reduction of $39 \%$. One patient at the lower initial dose also had SD and $13 \%$ reduction in tumor dimension. Although preliminary, the plasma pharmacokinetics of GTI-2040 determined a $\mathrm{C}_{\max }$ of $3.33 \mu \mathrm{g} / \mathrm{ml}$ and elimination $\mathrm{T}_{1 / 2}$ of $0.95 \mathrm{~h}$ at the phase II dose.

Clinical trials of GTI-2040 are currently being conducted by the NCI in the US and Canada. Based on the trend in antisense therapeutic development towards combination therapy with standard chemotherapeutic drugs and dramatic anti-tumor activity of GTI-2040 in combination with a number of agents in pre-clinical models of human cancer (Lee Y et al, AACR Special Conference in Cancer Research; Advances in Breast Cancer Research, 2003, and AACR Annual Meeting 2004), these trials were designed for combination treatments. These studies incorporate correlative science components to assess whether target down-regulation correlates with clinical outcome and whether the effect on RRM2 levels in WBCs correlates to RRM2 levels in tumor samples, and include a combination of GTI-2040 with capecitabine in metastatic breast cancer (current study), with gemcitabine in solid tumors, with oxaliplatin and capecitabine in metastatic colorectal cancer, with docetaxel in advanced non-small cell lung cancer and hormone refractory prostate cancer, and with cytarabine in acute myeloid leukemia.

An ongoing, CTEP-sponsored phase II trial of GTI-2040 in combination with capecitabine in metastatic breast cancer (NCI protocol \#6093; PHII-46) is being conducted by the California Cancer Consortium (University of California, Davis, City of Hope, University of Southern California, and University of Pittsburgh). Eligible patients include those with measurable disease that has progressed on at least one, but no more than two, prior regimens for metastatic disease. Patients receive a continuous intravenous infusion of GTI-2040 on days 1-14 and oral capecitabine twice daily on days 1-14 of a 21-day cycle. In this study, an assay for detecting changes in the expression of RRM2 and RRM1 mRNA after GTI-2040 antisense drug treatment was assessed in tumor tissue and WBC samples from 
Table I. Real-time PCR primers and probes.

\begin{tabular}{lllc}
\hline Gene & & \multicolumn{1}{c}{ Sequence } & \multicolumn{1}{c}{$\begin{array}{c}\text { GenBank } \\
\text { number }\end{array}$} \\
\hline B-actin & Forward: & 5'-CGAGCGCGGCTACAGCTT-3' & NM_00101 \\
& Reverse: & 5'-CCTTAATGTCACGCACGATT-3' & \\
& Probe: & 5'TET-ACCACCACGGCCGAGCGG-3'TAMRA \\
RRM1 & Forward: & 5'-CACTAGCTGCGATGCATGTGA-3' & NM_001033 \\
& Reverse: & 5'-TCGAGATGTAATTTTGCAAACATGA-3' & (222-295 bp) \\
& Probe: & 5'FAM-CAAGCGAGATGGCCGCCAAGAAC-3'TAMRA & \\
RRM2 & Forward: & 5'-ATGAAAACTTGGTGGAGCGATT-3' & NM_001034 \\
& Reverse: & 5'-TGGCAATTTGGAAGCATAGA-3' & (619-696 bp) \\
& Probe: & 5'FAM-CCAAGAAGTTCAGATTACAGAAGCCCGCTG-3'TAMRA & \\
TBP & Forward: & 5'-TTG TAT CCA CAG TGA ATC TTG GTT GT-3' & NM_003194 \\
& Reverse: & 5'-CTT GGG ATT ATA TTC GGC GTT T-3' & (735-817 bp) \\
& Probe: & 5'FAM-CTT GAC CTA AAG ACC ATT GCA CTT CG-3'TAMRA & \\
HuPo & Forward: & 5'TGG ACA GCG TGG GTA TCG A-3' & NM_001004 \\
(RPLP2) & Reverse: & 5'CTG GGC AAT GAC GTC TTC AA-3' & (156-247 bp) \\
& Probe: & 5'FAM-CGG ACG ACG ACC GGC TCA ACA-3'TAMRA & \\
18 S & Forward: & 5'-AACGAGACTCTGGCATGCTAACTA-3' & M10098 \\
& Reverse: & 5'-CGCCACTTGTCCCTCTAAGAA-3' & (1483-1562 bp) \\
& Probe: & 5'TET-TACGCGACCCCGAGCGGT-3'TAMRA &
\end{tabular}

Interim results for the L01-1409 study are from both the SN022 summary (pages 6 and 10) and the 2004 annual report. The NCI study information is summarized from the 2004 annual report, from information provided by Lorus. Current as of November 2003 - to be updated if Lorus has further data.

participating patients. The samples were collected prior to, and after treatment according to the protocol schedule. Total RNA was extracted and analyzed for mRNA expression of the ribonucleotide reductase subunits (RRM1 and RRM2) and for housekeeping genes (ß-actin, 18S, HuPo and TBP) by real-time PCR on the ABI 7900HT. The results suggest that this assay may be used to determine changes in target gene expression after treatment with GTI-2040. Furthermore, the ability to detect RRM2 in both WBC and tumor samples raises the question of whether WBCs can serve as an appropriate surrogate tissue for assessing target down-regulation.

\section{Materials and methods}

RNA isolation and cDNA synthesis. Human peripheral white blood cells (WBCs) were prepared from fresh blood samples drawn before and after administration of GTI-2040. The drug effect was assessed over a one-month period: the blood samples were collected before treatment (day 1), on day 2, 7 and 15 of the treatment cycle according to the schedule in the protocol. Erythrocytes were removed with Erythrocyte Lysis Buffer (Qiagen Inc., Valencia, CA) according to the manufacturer's instructions and WBC pellets were snap frozen and kept at $-80^{\circ} \mathrm{C}$ until processed further. Biopsy samples were kept frozen at $-80^{\circ} \mathrm{C}$ and disrupted with a Polytron homogenizer in the first step of RNA isolation. The total RNA was isolated using
RNeasy ${ }^{\circledR}$ Micro Kit (Qiagen Inc.) according to the manufacturer's instructions. Genomic DNA contamination was removed with DNase I treatment on the column. The integrity of isolated RNA was tested on 1\% agarose gel (SeaKem, FMC, Rockland, ME) or with Agilent 2100 Bioanalyzer. The RNA concentration and $A_{260}: A_{280}$ ratio was measured using a UV spectrophotometer.

cDNA was prepared from total RNA in a $25 \mu 1$ volume, using MMLV reverse transcriptase enzyme and random hexamers as primers (Invitrogen, Carlsbad, CA) as described previously (21-22). Quantification of gene expression was carried out using cDNA samples and a real-time PCR method. A set of primers and probes for each of TBP, RRM1 and RRM2 were purchased from Applied Biosystems, Foster City, CA (pre-designed Gene Expression Assays). Additional primers and probes for RRM1, RRM2 and B-actin were as described previously (21). 18S, TBP and HuPo primers and probes were designed according to the Applied Biosystems guidelines (Primer Express software, Applied Biosystems) to fit the realtime PCR requirements. Genomic DNA amplification was excluded by designing the primers around the exon-intron splicing sites. The sequences of primers and probes are shown in Table I.

Real-time PCR. Each PCR reaction was set up in a $20-\mu 1$ final volume containing $2 \mu \mathrm{l}$ cDNA from each sample and a 
A.

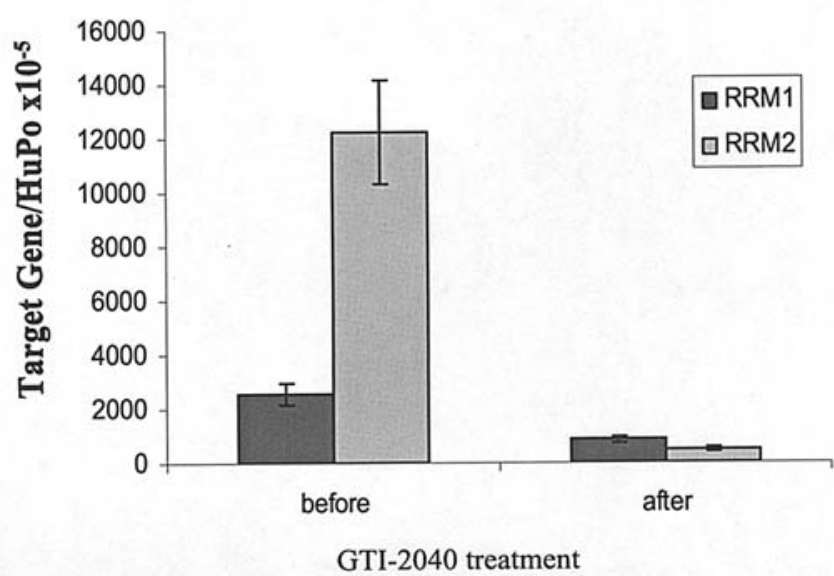

B.

Biopsy: HuPo normalized data

\begin{tabular}{lccc} 
& $\begin{array}{c}\mathrm{RRM} 1 \\
\times 10^{-5}\end{array}$ & $\begin{array}{c}\mathrm{RRM} 2 \\
\times 10^{-5}\end{array}$ & M2/M1 ratio \\
\hline before & $2592 \pm 404.5$ & $12245 \pm 1911.1$ & 4.73 \\
after & $868 \pm 110.5$ & $500 \pm 63.7$ & 0.58
\end{tabular}

Figure 1. Changes in RRM1 and RRM2 mRNA expression levels in patient biopsy samples before and after GTI-2040 treatment. We repeated the experiments with 2 different primer pairs and probes for the target genes. The arrow bars represent the SEM values from triplicate measurements. (A) RRM1 and RRM2 gene expression level in biopsy samples before and after the drug treatment normalized to human acidic ribosomal protein (HuPo) as a housekeeping gene. (B) The table shows the absolute values of RRM2 \& RRM1 expression level normalized to HuPo and the expression level ratio of the ribonucleotide reductase subunits in biopsy samples. SEM values were calculated from triplicate measurements. The RRM2:RRM1 ratio was calculated from the data normalized to HuPo (RPLP2).

TaqMan Universal PCR mix (Applied Biosystems). For target genes using in-lab designed primer/probe sets, the probe concentration was $0.3 \mu \mathrm{mol} / 1$ and primer concentrations for ß-actin, human acidic ribosomal protein (HuPo), TATA binding protein (TBP), RRM1 and RRM2 genes were $0.4 \mu \mathrm{mol} / 1$, and $0.2 \mu \mathrm{mol} / 1$ for the $18 \mathrm{~S}$ gene. For assays containing the ABI Gene Expression Assay primer/probe sets, $1 \mu 1$ primer and probe mix (20X concentration) was added to the reaction. The PCR amplification was performed on a 384-well plate using the default cycling conditions. During the PCR reaction, the TaqDNA polymerase cleaved the probe and released the 5 ' end reporter fluorescence dye (FAM, TET) whose fluorescence was detected by the ABI 7900HT Sequence Detection System (Applied Biosystems). For the absolute calibration curve of the target genes and the housekeeping genes, serial dilutions of the plasmid ( $10^{8}$ to 1 copy range) containing the gene insert were used. A cDNA sample from normal leukocyte total RNA (BD Biosciences, Palo Alto, CA) was used as positive control. Relative gene expression was determined as the ratio of the gene of interest to the internal reference gene expression based on the $\mathrm{Ct}$ values.

\section{Results}

Validation of the GTI-2040 inhibitory effect on RRM2 mRNA expression level. RRM1 and RRM2 mRNA expression levels were determined from patient white blood cells (WBCs), taken as part of their participation in the California Cancer Consortium phase II trial in metastatic breast cancer, by real-time PCR (TaqMan method). Whole blood samples were collected before and after the administration of GTI-2040. The drug effect was assessed on the 2nd or 3rd day after the treatment and on days 7 and 15 following the treatment. The data in this preliminary report are from a patient with metastatic breast cancer whose biopsy tissue samples were available with an observed clinical response of 6 months duration. As shown in Fig. 1A, there is a detectable 3-fold decrease in the level of RRM1 mRNA and an approximately 25-fold decrease in the RRM2 expression in the biopsy sample one day after GTI-2040 treatment. Fig. 1B shows that the RRM2/RRM1 ratio drops from 4.73 to 0.58 after treatment.

Fig. 2A shows the change in RRM1 and RRM2 gene expression in the WBC samples up to 15 days after GTI-2040 drug administration. The change in the RRM2 expression level was dramatic with an approximate 20 -fold decrease on day 2 following treatment. The expression level was $17 \%$ of the before treatment value on day 15 before the 7 day rest from therapy. No changes were detected in RRM1 mRNA level during the first week after treatment but the expression level had increased by the end of the 2nd week compared to the level before treatment. Fig. 2B shows changes in the RRM2:RRM1 ratio, with the same trend as changes in the absolute values; the ratio dropped from 4.73 to 0.24 on the 2 nd day after treatment and slowly increased to 0.78 by the 7 th day and dropped to 0.28 on day 15 after drug administration. Analysis of more patient samples is required to determine if this pattern of response to GTI-2040 is consistent.

\section{Discussion}

This study presents preliminary data regarding quantitation of RRM2 mRNA in the tumor and WBCs from a patient receiving GTI-2040 in combination with capecitabine for metastatic breast cancer. Four different housekeeping genes (ß-actin, 18S, HuPo and TBP) were used as internal references to normalize the data. This was essential to determine whether the expression of a chosen housekeeping gene is changing under the experimental conditions (23). Appropriate validation of internal references is crucial to avoid misinterpretations of study findings (24). It has become clear that $B$-actin and 


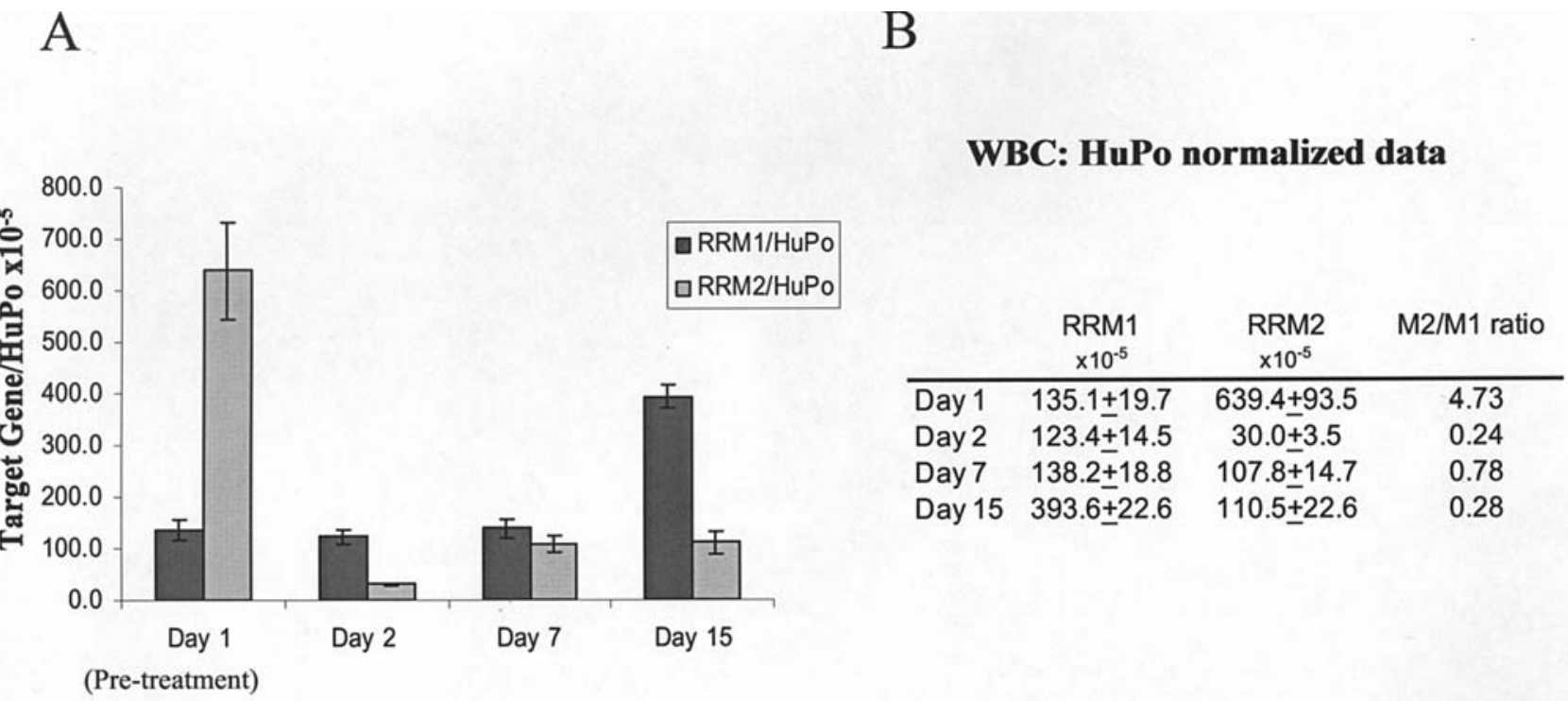

\section{GTI-2040 treatment}

Figure 2. Changes in RRM1 and RRM2 mRNA expression levels in patient WBC samples before and after GTI-2040 treatment. (A) Changes in RRM1 and RRM2 mRNA expression level from patient WBC samples before and after GTI-2040 treatment. The results were normalized to HuPo as a housekeeping gene. The arrow bars represent the SEM values from triplicate measurements. (B) The table shows changes in the expression of the two subunits (RRM2, RRM1) absolute values from patient WBCs normalized to HuPo, changes in the RRM2:RRM1 ratio after treatment and standard SEM values from triplicate measurements.

glyceraldehydes-3-phosphate-dehydrogenase (GAPDH) may be inappropriate as internal references because of their variability (25). $18 \mathrm{~S}$ gene expression is extremely high and as a result, amplification is not in the same range of expression as the target genes in this study. The reliability of HuPo and TBP as reference genes to study changes in gene expression for GTI-2040 clinical trials (data not shown) in our studies are in agreement with published results (24). HuPo was chosen for normalization of expression data because it is a more abundant gene than TBP and the data was in the range for more reliable measurement of $\mathrm{Ct}$ values. The results in Figs. 1 and 2 show that the method can detect changes in RRM2 mRNA expression levels during GTI-2040 treatment from the patient's biopsy and blood samples and that this is a reliable real-time PCR method.

Although the tumor/WBC paired analysis in this report is restricted to samples from a single patient, the observed decrease in mRNA levels in both biopsy and WBC samples from a responding patient (Figs. 1 and 2) is compelling. With an increasing number of patient samples analyzed in this ongoing trial, the extent of a correlation between changes in RRM2 levels and clinical response will become evident, as will the statistical significance of any observed correlation. Although there have been only a few patient samples analyzed to date, preliminary data suggest that there are inter-patient differences in the baseline levels of RRM2 in WBCs prior to therapy. Thus, correlation of baseline RRM2 mRNA levels with the ability of GTI-2040 to down-regulate the target and subsequent patient clinical outcomes will be of considerable interest. Finally, as more tumor tissue samples are available, how changes in RRM2 levels in WBCs correlate with tumor tissue will become apparent and will determine whether WBCs are an acceptable surrogate tissue for approximating target down-regulation in the target tissue.
Although this trial and other NCI sponsored clinical trials are evaluating GTI-2040 in combination with approved chemotherapeutics, there is little information regarding the pharmacokinetics of these drug combinations in humans. Equally important to correlation of changes in RRM2 with clinical response will be comparison of pharmacokinetic profiles with clinical response and target down-regulation. While the results of our ongoing studies should be considered exploratory, these collective data may provide an indication of the dose optimization of GTI-2040 and determination of sequence specificity in combination with chemotherapy.

In conclusion, a fast and reliable method for quantifying changes in target gene expression during treatment with GTI-2040 has been developed. This real-time RT-PCR assay has the potential to assess target down-regulation by GTI-2040 in a clinical setting.

\section{Acknowledgements}

We thank Christina Qi for the excellent technical work, Eloise Lopez-Leuvason, R.N. for the collection of clinical samples, and Viengthong Chai, Peter Murray, Dr Yoon Lee, Dr Edward Newman and Jennifer Shih for the critical review of the manuscript. We thank Dr Jim A. Wright and Dr Aiping Young for their support of this work. This study was supported in part by N01-CM 70701, SAIC-Frederick contract 24XS034, 24XS037, 24XS065 and NCI-72767 (Y. Yen).

\section{References}

1. Eklund H, Uhlin U, Farnegardh M, et al: Structure and function of the radical enzyme ribonucleotide reductase. Prog Biophys Mol Biol 77: 177-268, 2001.

2. Nocentini G: Ribonucleotide reductase inhibitors: new strategies for cancer chemotherapy. Crit Rev Oncol Hematol 22: 89-126, 1996. 
3. Chabes A, Georgieva B, Domkin V, et al: Survival of DNA damage in yeast directly depends on increased dNTP levels allowed by relaxed feedback inhibition of ribonucleotide reductase. Cell 112: 391-401, 2003.

4. Fritzer-Szekeres M, Luxbacher C, Horvath Z, et al: Apoptosisinducing cleavage of caspases by trimidox, an inhibitor of ribonucleotide reductase. Adv Exp Med Biol 486: 125-130, 2000.

5. Fritzer-Szekeres M, Salamon A, Grusch M, et al: Trimidox, an inhibitor of ribonucleotide reductase, synergistically enhances the inhibition of colony formation by Ara-C in HL-60 human promyelocytic leukemia cells. Biochem Pharmacol 64: 481-485, 2002.

6. Fritzer-Szekeres M, Grusch M, Luxbacher C, et al: Trimidox, an inhibitor of ribonucleotide reductase, induces apoptosis and activates caspases in HL-60 promyelocytic leukemia cells. Exp Hematol 28: 924-930, 2000

7. Hakimelahi GH, Moosavi-Movahedi AA, Sambaiah T, et al: Reactions of purines-containing butenolides with L-cysteine or $\mathrm{N}$-acetyl-L-cysteine as model biological nucleophiles: a potent mechanism-based inhibitor of ribonucleotide reductase caused apoptosis in breast carcinoma MCF7 cells. Eur J Med Chem 37: 207-217, 2002.

8. Rosenberger G, Fuhrmann F, Grusch M, et al: The ribonucleotide reductase inhibitor trimidox induces c-myc and apoptosis of human ovarian carcinoma cells. Life Sci 67: 3131-3142, 2000.

9. Fan H, Villegas C, Huang A and Wright JA: The mammalian ribonucleotide reductase $\mathrm{R} 2$ component cooperates with a variety of oncogenes in mechanism of cellular transformation. Cancer Res 58: 1650-1653, 1998.

10. Fan H, Villegas C and Wright JA: Ribonucleotide reductase R2 component is a novel malignancy determinant that cooperates with activated oncogenes to determine transformation and malignant potential. Proc Natl Acad Sci USA 93: 14036-14040, 1996.

11. Huang A, Fan H, Taylor WR and Wright JA: Ribonucleotide reductase R2 gene expression and changes in drug sensitivity and genome stability. Cancer Res 57: 4876-4881, 1997.

12. Lin ZP, Belcourt MF, Cory JG and Sartorelli AC: Stable suppression of the R2 subunit of ribonucleotide reductase by R2-targeted short interference RNA sensitizes p53(-/-) HCT-116 colon cancer cells to DNA-damaging agents and ribonucleotide reductase inhibitors. J Biol Chem 279: 27030-27038, 2004.

13. Duxbury MS, Ito H, Benoit E, et al: Retrovirally mediated RNA interference targeting the M2 subunit of ribonucleotide reductase: a novel therapeutic strategy in pancreatic cancer. Surgery 136: $261-269,2004$
14. Duxbury MS, Ito $\mathrm{H}$, Zinner MJ, et al: RNA interference targeting the M2 subunit of ribonucleotide reductase enhances pancreatic adenocarcinoma chemosensitivity to gemcitabine. Oncogene 23: $1539-1548,2004$

15. Orr RM: Lorus Therapeutics. Curr Opin Invest Drugs 2: 1462-1466, 2001.

16. Lee Y, Vassilakos A, Feng N, et al: GTI-2040, an antisense agent targeting the small subunit component (R2) of human ribonucleotide reductase, shows potent antitumor activity against a variety of tumors. Cancer Res 63: 2802-2811, 2003.

17. Levin AA: A review of the issues in the pharmacokinetics and toxicology of phosphorothioate antisense oligonucleotides. Biochim Biophys Acta 1489: 69-84, 1999.

18. Jason TL, Koropatnick J and Berg RW: Toxicology of antisense therapeutics. Toxicol Appl Pharmacol 201: 66-83, 2004.

19. Desai AA, Schilsky RL, Young A, et al: A Phase I study of antisense oligonucleotide GTI-2040 given by continuous intravenous infusion in patients with advanced solid tumors. Ann Oncol 16: 958-965, 2005

20. Desai RB, Murray P, Poiesz B, Quinn D, et al: Interim evaluation of a multi-institute phase I/II study of antisense oligonucleotide GTI-2040 (G) and capecitabine (C) in patients with metastatic renal cell carcinoma (mRCC). Eur J Cancer 2 (suppl): S136, 2004.

21. Juhasz A, Frankel P, Cheng C, et al: Quantification of chemotherapeutic target gene mRNA expression in human breast cancer biopsies: comparison of real-time reverse transcription-PCR vs. relative quantification reverse transcription-PCR utilizing DNA sequencer analysis of PCR products. J Clin Lab Anal 17: 184-194, 2003.

22. Chai V, Vassilakos A, Lee Y, Wright JA and Young AH: Optimization of the PAXgeneTM blood RNA extraction system for gene expression analysis of clinical samples. J Clin Lab Anal 19: 182-188, 2005.

23. Eads CA, Danenberg K, Kawakami K, et al: CpG island hypermethylation in human colorectal tumors is not associated with DNA methyltransferase overexpression. Cancer Res 59: 2302-2306, 1999.

24. Dheda K, Huggett JF, Bustin SA, et al: Validation of housekeeping genes for normalizing RNA expression in real-time PCR. Biotechniques 37: 112-119, 2004.

25. Bustin SA: Quantification of mRNA using real-time reverse transcription PCR (RT-PCR): trends and problems. J Mol Endocrinol 1: 23-39, 2002. 